FERNANDO PORTAL CARRASCO

ESCUELA DE ARQUITECTURA

FACULTAD DE ARQUITECTURA,

DISEÑO Y ESTUDIOS URBANOS

PONTIFICIA UNIVERSIDAD CATÓLICA DE CHILE

SANTIAGO, CHILE

FPORTAL@UC.CL
Fecha de recepción: 08/03/2015

Fecha de aceptación: 12/04/2016

Cómo citar: Portal Carrasco, F. (2016). Diseño

y desarrollo en las periferias del capitalismo.

Transferencias metodológicas entre HfG-UIm

y el Grupo de Diseño del INTEC. RChD: creación

y pensamiento, $1(1)$, 37-51.

DOI: 10.5354/0718-2430.2016.44199

Revista Chilena de Diseño

RChD: creación y pensamiento

Universidad de Chile

2016, 1(1)

http://rchd.uchile.cl

\section{Diseño y desarrollo en las periferias del capitalismo. Transferencias metodológicas entre HfG-Ulm y el Grupo de Diseño del INTEC}

\author{
Design and Development in Capitalism Peripheries. Methodological \\ Transferences Between HfG-UIm and INTEC Design Group
}

Resumen. El presente texto presenta las inquietudes iniciales de una investigación (CNCA Fondart 2015), que tiene como objetivo el documentar, analizar y difundir la producción del Grupo de Diseño Industrial, liderado por Gui Bonsiepe, y alojado en el Comité de Investigaciones Tecnológicas (INTEC) de CORFO, entre enero de 1970 y septiembre de 1973, durante la presidencia marxista de Salvador Allende. La investigación busca profundizar en las condiciones de desarrollo disciplinar del diseño industrial en América Latina y en Chile, a través del análisis de los fenómenos de transferencia, adaptación, apropiación y transformación de la metodología disciplinar desarrollada en HfG-Ulm. La hipótesis de trabajo sostiene que a través del análisis de los documentos, y de la reconstrucción analítica de los prototipos generados por el Grupo de Diseño, se podrá reconocer, comprobar y avanzar en el análisis de sus metodologías proyectuales, entendidas como producto de las condiciones culturales y políticas del período, asociadas al rol del diseño en sociedades no competitivas, y a la búsqueda proyectual de una independencia tecnológica.

Palabras clave: funcionalismo, HfG-Ulm, INTEC, investigación en diseño, metodologías de diseño, postguerra.

\begin{abstract}
This paper constitutes the starting point of a research (CNCA Fondart 2015) oriented towards the documentation, analysis and dissemination of the production of the Industrial Design Group, a governmental team lead by Gui Bonsiepe, active within the Technological Research Committee (INTEC) of the Chilean Economic Development Agency (CORFO), between January 1970 and September 1973, during the Marxist presidency of Salvador Allende. The research aims to deepen the study of design's history in Latin America and Chile, by analysing processes of transference, adaptation, appropriation and transformation of design methods coined in post-war Europe, and specifically by HfG-Ulm. The working hypothesis states that the compiling of the Design Group's archive and the analytical reconstruction of its prototypes would allow determining the late development of these project methodologies, in a cultural and political context defined by a non-competitive society and the national search for technological independence.
\end{abstract} Keywords: design methodology, design research, funcionalism, HfG-Ulm, INTEC, post-war. 
Figura 1. El 4 de mayo de 1968, en la inauguración de una exposición sobre la Bauhaus, protestantes en contra del cierre de HfG-UIm se reúnen con pancartas que dicen: "Ilorar por la Bauhaus, proteger la $\mathrm{Hfc}^{\prime}$, "las palabras sobre la Bauhaus son buenas, las acciones sobre la $\mathrm{HfC}$ son mejores", y "en 1968, enterrada durante la excavación de la Bauhaus: la HfG".

Fuente: Archivo Südwestpresse, en Spitz (2002).

1. La categoría de "teoría crítica de la Hfc" es acuñada por Kenneth Frampton (1974) en su relato sobre la escuela, para denominar las líneas comunes de pensamiento teórico desarrolladas por Tomás Maldonado, Abraham Moles, Claude Schnaid y Gui Bonsiepe. Esta teoría crítica se encontraba en contraposición a aproximaciones más flexibles hacia la relación entre diseño y consumo, las cuales pueden encontrarse en las posiciones de otros profesores como Otl Archer y Anthony Froshaug. Una clara declaración de la teoría crítica está enunciada en un conjunto de tres ensayos publicados por estos autores en el número 19/20 del Journal of the Ulm School of Design (Bonsiepe, 1967; Moles, 1967; Schnaid, 1967).

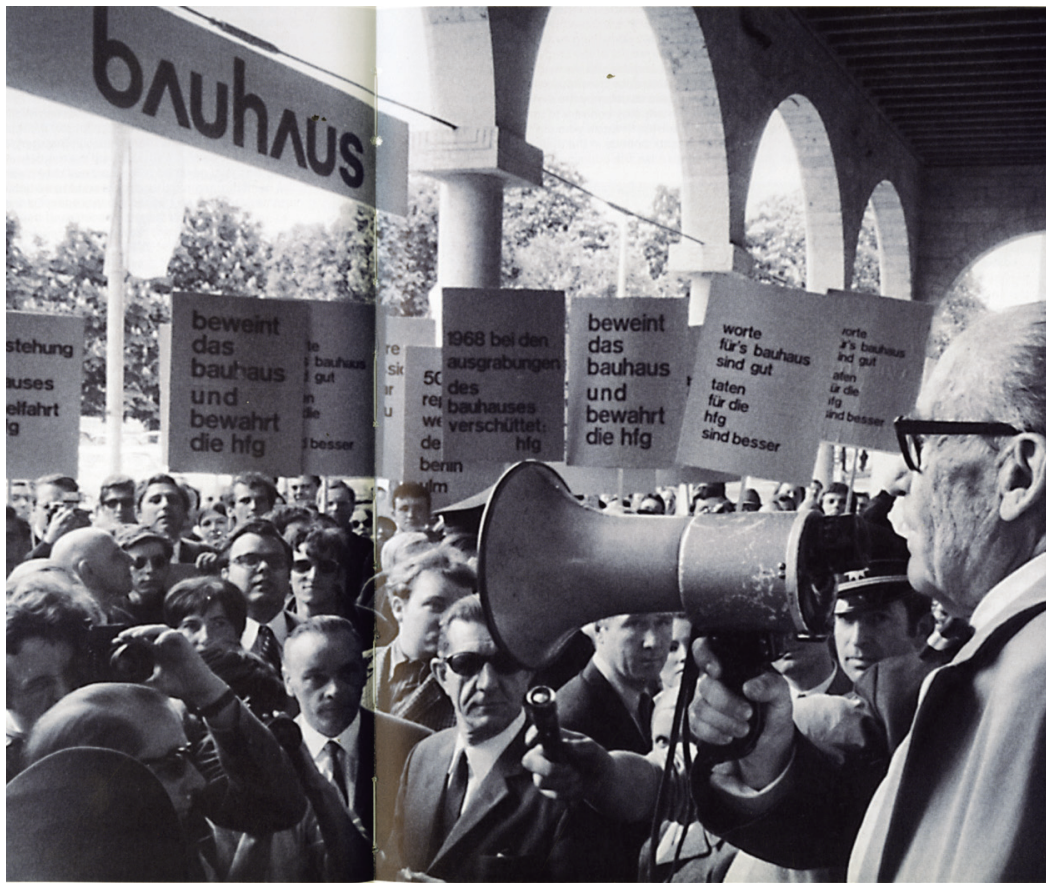

\section{Introducción}

El 30 de septiembre de 1968, HfG-Ulm dio por terminadas sus actividades. Su cierre marcó el fin de un proceso de intervención de la escuela y de su programa, el cual implicó el retiro de financiamiento por parte de la autoridad local y la puesta en riesgo de su autonomía institucional. Esta intervención, marcó también un giro en la formación de un discurso crítico sobre el diseño industrial en la escuela iniciado por el programa del primer rectorado de Tomás Maldonado de 1957.

Sin embargo, su cierre no significó el fin de este proceso, sino su transferencia a otros ámbitos de ejercicio y comprobación. La diáspora que siguió al cierre forzó la manifestación de los conflictos políticos que habían sido previstos para la liberación de la práctica del diseño, provocando un traslado de su teoría crítica ${ }^{1}$ desde un plano conceptual a un terreno práctico. Durante los últimos años de la escuela, esta teoría cuestionaba el rol profesional del diseño en el proceso capitalista de mercantilización y su instrumentalización como herramienta de control hegemónico. Este discurso fue desarrollado por un conjunto de profesores, incluyendo a Tomás Maldonado, Gui Bonsiepe, Abraham Moles y Claude Schnaid, a través de una serie de cursos, artículos, charlas, colaboraciones y proyectos.

En ellos, la conformación del diseño industrial -entendido como una profesión orientada hacia la intervención de la cultura, a través de su diseño material y del diseño de sus artefactos semánticos - se encontraba frente a un dilema paralizante cuyas únicas alternativas implicaban la redefinición de la profesión - enfrentada a los modelos capitalistas de producción-, o la redefinición de estos modelos - pasando de una sociedad competitiva a una no competitiva. Dado que el cierre de la escuela pareciera dar constancia de los límites de la primera opción, revisar los alcances de la segunda opción implica aproximarse de manera crítica al devenir de las ideas de HfG-Ulm en el contexto de las sociedades no competitivas del período. 


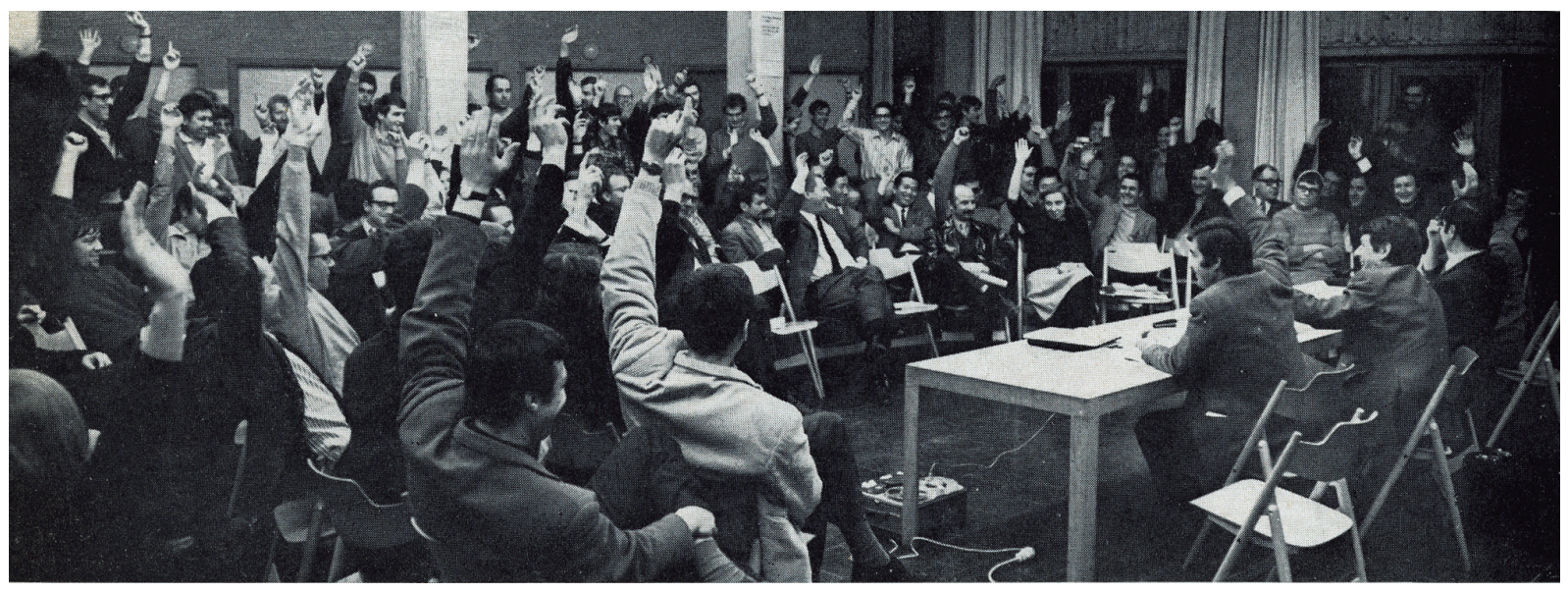

Para este objetivo, la práctica profesional de Gui Bonsiepe, una vez cerrada la escuela, se transforma en una experiencia especialmente relevante. Su inmediata partida a Chile en octubre de 1968 lo lleva a involucrarse directamente en la definición material y semántica de una sociedad no competitiva, por medio de su trabajo en el Grupo de Diseño del Comité de Investigaciones Tecnológicas de CORFO (INTEC), el cual dirigió en el marco del gobierno del presidente Salvador Allende.

En esta experiencia, es posible leer un diálogo entre las posibilidades y preocupaciones planteadas por los miembros de la Escuela de Ulm, y su transformación desde su implementación en una sociedad no competitiva. Unos años antes, en 1965 -y a través de su colaboración en The Education of Vision² de Gyorgy Kepes (1965)-, Tomás Maldonado dedicó una sección completa de su artículo Design Education al tema del Diseño en una sociedad no competitiva, donde afirma:

[...] la particular orientación que la conformación del diseño industrial está tomando en países socialistas, donde la estructura económica y social es diferente a la nuestra [...] puede servir para responder muchas preguntas, y para clarificar nuestro propio pensamiento sobre la profesión del diseñador industrial. ¿Es esta una profesión a ser considerada como una actividad justificable solo dentro de una sociedad competitiva?, o ¿la función realizada por el diseñador industrial es esencialmente la misma en una economía donde la competición tiende a desaparecer o donde ya ha desaparecido? (Maldonado, 1965)

A través de estas preguntas, Maldonado invita a su audiencia estadounidense a concebir la actividad del diseño como algo distinto del marketing profesional y del conservadurismo académico del good design. Y lo hace presentando para este contexto, nuevos horizontes:

Más que la imitación de nuestras debilidades, esperamos que los diseñadores en sociedades no competitivas exploten sus propias posibilidades. Esperamos que aborden problemas que nosotros no podemos abordar. Por ejemplo, los productos técnicos requieren la revisión urgente de sus propiedades

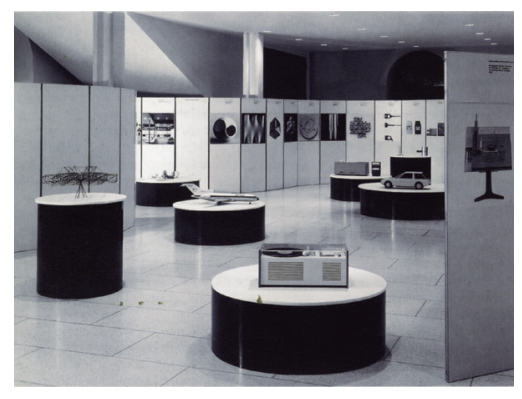

Figura 2. Votación 23 de febrero, 1968, en la resolución por cerrar la Escuela.

Fuente: Archivo HfG-Ulm, en Bonsiepe (2010, p.8). Figura 3. Fotografía de la inauguración de la exposición de Hfc en Kornhaus of Ulm, I de octubre, 1963, mostrando los diferentes productos diseñados por los talleres de la Escuela. Fuente: Archivo HfG-Ulm (63/0461), en Spitz (2002).

2. Este libro fue el primero de los siete volúmenes de la serie Vision + Value editado por Kepes en MIT. Esta publicación siguió al catálogo de la exposición The New Landscape in Art and Science. 
3. Los 21 números de la revista de la escuela corresponden a dos proyectos editoriales consecutivos. En palabras de Bonsiepe: "La historia de esta publicación puede ser dividida en dos fases, que difieren no solo en términos de la presentación gráfica - desde un formato poco cómodo rectangular hasta un formato $\mathrm{A}_{4}$ con el diseño de Tomás Gonda-, sino también en su concepto editorial. La primera fase se extendió desde octubre de 1958 hasta julio de 1959 (números del 1 al 5), y la segunda fase desde octubre de 1962 hasta marzo de 1968 (números del 6 al 21). Inicialmente la dirección de la escuela era responsable por el contenido. Luego se formó un consejo editorial separado, compuesto por tres personas: Tomás Maldonado, la secretaria de redacción Renate Kietzmann y el autor de este artículo. Esto no significaba que solo dos o tres personas decidían qué sería publicado; todos los docentes eran exhortados a presentar textos y materiales para mostrar el nivel de los resultados alcanzados en su trabajo y sus tomas de posición. El proceso complicado y muchas veces contraproducente del referato anónimo no era observado; el equipo editorial no se veía en la obligación de llevar a cabo este ritual académico, con sus ventajas y desventajas" (Bonsiepe, 2006)

4. Vale la pena mencionar que este artículo es la transcripción de una lectura dictada por Bonsiepe en Buenos Aires en junio de 1966, en el Instituto Tecnológico Industrial (INTI). La relación entre la HfG y las escuelas y prácticas del diseño incipiente en Argentina -además de la anterior influencia de Maldonado en Nueva Visión- involucran la visita del Comité Argentino a la HfG-Ulm en 1962, el que más tarde fundará el Centro de Investigaciones de Diseño Industrial (CIDI), dependiente del INTI. La colaboración entre HfG y CIDI puede ser encontrada en Fernández (2006). Cabe destacar también que a esta charla de Bonsiepe en Buenos Aires, asisten entre otros Rodrigo Walker, y el equipo de estudiantes de diseño de la Universidad de Chile, con lo que Bonsiepe trabajará a su llegada a Santiago y que más tarde conformarán el Grupo de Diseño Industrial del INTEC. estructurales y funcionales. Pero en nuestra sociedad competitiva iniciativas de este tipo no se desarrollan, dado que nuestra principal actividad es el marketing de estos productos, eliminándose cualquier intento por cuestionar su artificiosa estabilidad. En una sociedad no competitiva, los diseñadores están en una posición favorable para enfrentar este tipo de tareas, pero hasta el minuto, no se ha avanzado mucho. (Maldonado, 1965)

Desde este punto de vista, revisar la experiencia de Bonsiepe como diseñador en una sociedad no competitiva permite revisar la negociación efectuada entre un programa crítico que apuntaba a "desestabilizar el carácter artificial y estable de los productos" (Maldonado, 1965) y la naturaleza específica de la conformación profesional del diseño.

\section{El proyecto de diseño de Gui Bonsipe y la HfG-Ulm}

Como parte del comité editorial del Journal of the Ulm School of Design, 3 desde 1962 hasta su último número en marzo de 1968, Bonsiepe jugó un rol preponderante en la definición del discurso de la escuela. Entre las distintas posiciones enfrentadas, su posición estuvo fuertemente alineada con el desarrollo de una teoría crítica del diseño. Esta teoría encontraba sus bases en el reconocimiento de la contradicción entre funcionalismo y producción orientada al consumo y en el entendimiento del potencial represivo de la estética al servicio del poder. Estas paradojas fueron directamente tratadas en sus contribuciones al último número de la revista. La revisión de estos textos - publicados en agosto de 1967- nos permitirán entender su posición al momento de llegar a Chile en octubre de 1968.

El primer artículo, Arabesques of Rationality, Notes on the Methodology of Design (Bonsiepe, 1967), ${ }^{4}$ revisa las principales tendencias contemporáneas de la definición de un método de diseño adecuado para el campo del diseño industrial. Frente al reconocimiento de que "una profesión orientada hacia la tecnología y la industria, difícilmente escapará de la ducha fría del cientificismo y la racionalización" (Bonsiepe, 1967, p.9), Bonsiepe presenta como inevitable la introducción de métodos racionales en la práctica del diseño. Aún así, mantiene también una posición imparcial frente a la gran cantidad de métodos a considerar: más que abogar por un método en particular, el artículo presenta distintos sistemas de toma racional de decisiones. Sistemas que si bien ya habían sido introducidos en la práctica y debate del diseño, tenían su origen en disciplinas externas como la ingeniería, la gestión, el marketing, las ciencias básicas y la planificación.

A lo largo de esta revisión, Bonsiepe insiste en evitar metodologías extremadamente racionales, y en la necesidad del diseño de desarrollar sus propios métodos:

Hoy la metodología del diseño está en la misma posición que la psicología en el siglo XIX, cuando anhelaba el estatus de una ciencia "de verdad". El método científico, sigue siendo el ídolo del cientifismo. Nosotros debemos preocuparnos de que el diseño no se someta a un ideal metodológico heteronómico, en el cual puede prácticamente anularse. Solo cuando la metodología del diseño se libere a sí misma de su frecuente relación parasitaria con otras disciplinas, podrá llegar a una estado superior. (Bonsiepe, 1967, p.23) 


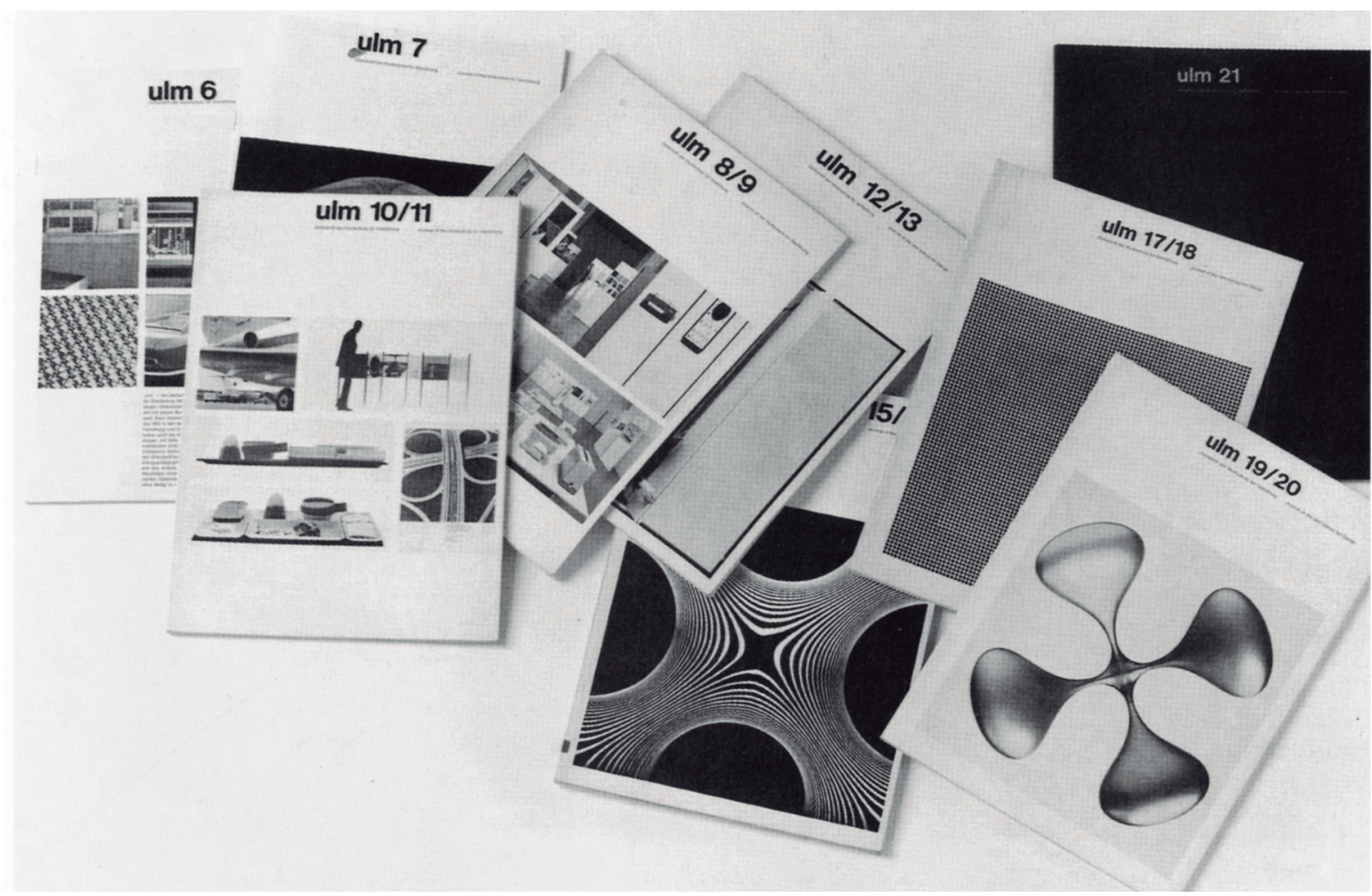

Frente a este prematuro estado del diseño y su disposición a adoptar metodologías en forma parasitaria, Bonsiepe enfatiza las prácticas de diseño e ingeniería como los puntos críticos de la transformación científica del diseño. En el último número de la revista, Bonsiepe toma la voz editorial en una serie de artículos y comentarios relacionados con el cierre de la escuela. El argumento enfatizado dentro de esta secuencia incluye la presentación del programa y una caracterización de los cambios con los que la profesión del diseño se ha encontrado desde su fundación:

Mientras que en teoría, el diseñador debía ser el representante y el intérprete del interés de la sociedad como un todo, el diseño se ha transformado en un elemento estratégico de las políticas empresariales modernas, en las cuales la competencia por el precio, ha sido reemplazada por la diferenciación de los productos, desde el diseño.(Bonsiepe, 1968b, p.9)

Denunciar la cooptación del diseño por parte de los agentes económicos que definen una sociedad basada en el consumo es un punto de partida común para algunos artículos de esta teoría crítica. ${ }^{5}$ En este discurso, el cambio en el rol social del diseño es entendido como una consecuencia del establecimiento de nuevos valores, propios de una "concepción cultural, enfocada en el cultivo de individuos económicamente independientes, donde los aspectos sociales son ignorados" (Bonsiepe, 1968b, p.5).

Este argumento surge como reacción ante las consecuencias culturales de la reconstrucción económica de Alemania, un proceso del cual la escuela fue
Figura 4. Journal of the UIm School of Design. Fuente: Archivo HfG-Ulm, en Lindinger (1991).

5. Este discurso también puede ser encontrado en la relación que Tomás Maldonado establece en Design and Sales, en Maldonado (1965, p.125). Como también en Moles (1967). 
un agente por al menos 20 años, y que transfiere los objetivos del diseño desde su provisión básica, hacia su consumo continuo. Al respecto, en su artículo Communication and Power, Bonsiepe declara:

Antes, la estética representaba la anticipación de nuestro estado de situación, permitiéndonos liberarnos de las restricciones impuestas por la necesidad. Pero la estética se encontró con un destino imprevisto. Se descubrió que de forma simple, podía ser puesta al servicio de la represión. Las formas del poder se sublimaron. En el transcurso de esta sublimación, la estética - que era y que aún contiene la promesa de liberar a la humanidad- ha sido cooptada por las fuerzas de la represión, y como tal, está siendo usada para obtener y conservar el poder. (Bonsiepe, 1968c, p.16)

Apenas siete meses después de esta publicación, Bonsiepe llega a Chile trayendo consigo, no solo una posición frente al diseño, sino también la búsqueda por establecer nuevas relaciones entre el diseño y el poder, en el contexto de una práctica estética orientada socialmente.

\section{La sociedad no competitiva de la Unidad Popular}

Tras esto, los miembros de HfG-Ulm están buscando la posibilidad de trabajar, lo cual quiere decir, una nueva atmósfera donde trabajar, que les permita no solo aceptar la sobrevivencia bajo la imposición de conceptos ajenos, sino vivir de acuerdo con sus propias ideas. Los miembros de HfG-Ulm traerán consigo ideas y experiencias de una institución que ha sido llamada una de las mejores y más progresistas escuelas de diseño del mundo. Queremos trabajar en el campo del diseño de productos, cine, diseño visual, comunicaciones, arquitectura, y de la preparación de la ciencia del diseño.

Nosotros preguntamos:

¿Qué individuo

¿Qué institución

¿Qué compañía

¿Qué ciudad

¿Qué gobierno

le dará residencia a $\mathrm{HfC}$ ? Una residencia que les permita ser productivos, y continuar lo que ha sido comenzado de forma tan promisora.

Por favor contactar:

Hochschule für Gestaltung 79 UIm Postlach 362 Germany. (Traducción anuncio Figura 5).

Una de las instituciones que respondió este llamado fue la Organización Internacional del Trabajo (OIT), agencia de desarrollo dependiente de las Naciones Unidas. Bonsiepe fue contratado por la oIT para participar del desarrollo de programas para el fomento de la pequeña y mediana industria en Chile. Actuando como consultor del Servicio de Cooperación Técnica (Sercotec), perteneciente a la Corporación del Fomento de la Producción (CORFO). Durante los últimos dos años del gobierno Demócrata Cristiano de Eduardo 

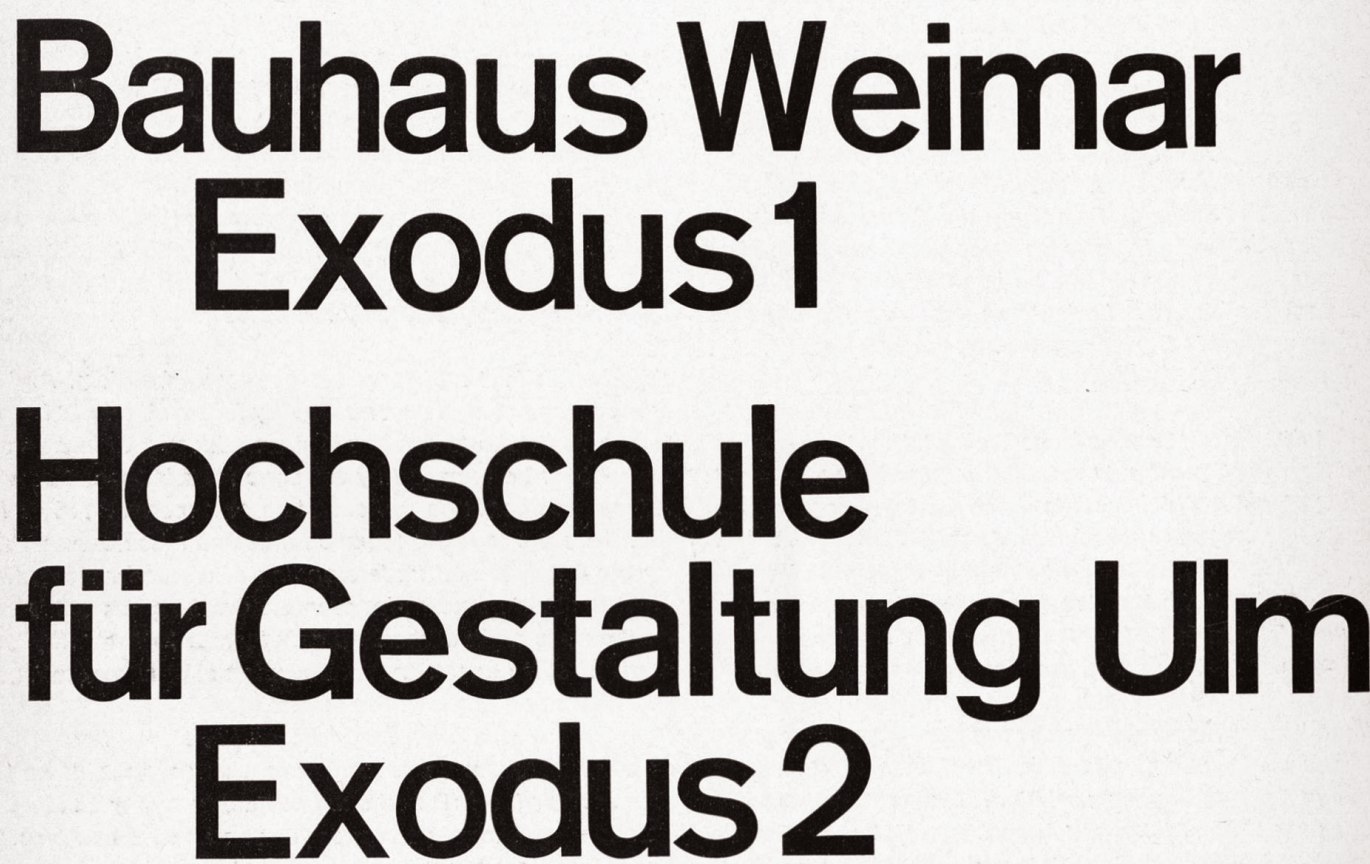

Even before the beginning of the Nazi regime the existence of the Bauhaus people in Weimar was put under press and finally made impossible. They drew the only conclusion: they left.

In Dessau they found a new home and a more apt environment to continue their work.

Th a similar to the one faced by the Bauhaus. Those employed at the $\mathrm{HfG}$ were informed on Monday, 19th Feb 1968 that on the 30th of September 1968 their contracts would be terminated. They have to (and they are allowed to) seek other activities elsewhere. Several proposals have been declared as to how the HfG might be carried on; preservation, rescue, consolidation and fusion with another institution under a cover association have been spoken of, but whatever the details of these plans, one point spoken but what will be left of the remains evident: What will be left of the Hect
Therefore members of the HfG UIm are looking for a chance to work, that is to say, a new atmosphere to work in, which enables them not only to accept survival under imposed alien concepts but to live according to their own ideas.

The members of the $\mathrm{HfG}$ will bring with them ideas and experiences from an institution which has been called one of the best and most progressive design schools in the world. We want to work in the field of product design, film, visual communications, architecture and prepare a science of design. We ask:

which individual

which institution

which company

which city

which government

will grant the $\mathrm{HFG}$ residence? A residence which would enable

them to be productive, and to continue what began so promisingly.

Please contact:

Hochschule für Gestaltung 79 Ulm Postfach 362 Germany

19 Feb 1968

Teachers and Students of the Hochschule für Gestaltung

Figura 5. Anuncio incluido en el último número de la revista Journal of the UIm School of Design.

Fuente: Archivo HfG-Ulm, en Bonsiepe (2010, p.8) 
6. "En la síntesis de la labor realizada por la CORFO entre 1964 y 1970 es necesario poner de relieve el Programa de Fomento de las Actividades de la Pequeña Industria y de la Artesanía Nacional, encargado al Servicio de Cooperación Técnica y puesto en marcha con aportes del Banco Interamericano de Desarrollo y de las Naciones Unidas, más los expresamente asignados por la Corporación. La acción de Sercotec se centró en la aplicación y difusión tanto de los principios como de las técnicas de administración de empresas en cuanto a organización general, personal, finanzas, producción y comercialización. Esta labor contempló asesorías técnicas a diversas entidades públicas y privadas, cursos de adiestramiento y fomento de la producción a través de proyectos sectoriales". En Ortega, Norambuena, Pinto, y Bravo (1989), citado en Palmarola (2002).

7. Una breve reseña de las relaciones entre la diáspora de la HfG-Ulm y el establecimiento de la educación en diseño en América Latina, puede encontrarse en Fernández (2006).
Frei Montalva, Bonsiepe desarrolló una serie de proyectos, incluyendo envases de comestibles, juguetes y muebles de cocina para la vivienda básica. ${ }^{6}$ También estuvo en contacto con las jóvenes escuelas de diseño fundadas recientemente en Chile, y con la movilización estudiantil de la reforma. ${ }^{7}$ Tras la primera elección democrática de un gobierno marxista en 1970, el gobierno de Salvador Allende (1970-1973) desarrolló -bajo la promesa de una vía pacífica hacia el socialismo-, un proceso de nacionalización empresarial sin precedentes. Por medio de la nacionalización de los medios de producción, el Estado esperaba tomar control de la industria, y del mercado, desde ambos lados, de la oferta y la demanda.

Dentro de este proceso de transformación de una sociedad competitiva en una no competitiva, Bonsiepe es invitado por Fernando Flores -en aquel entonces Subgerente Técnico de CORFO-, a dirigir el Grupo de Diseño Industrial del Instituto de Investigaciones Tecnológicas (INTEC).

El grupo estaba orientado a desarrollar, a través de la investigación aplicada, una serie de proyectos conducentes al establecimiento de una relación directa entre diseño, tecnología, industria, innovación y desarrollo. Los objetivos principales del Grupo de Diseño estaban alineados con la estrategia de desarrollo de sustitución de importaciones -común en la región durante los sesenta y setenta-, la cual en el caso chileno, se encontraba radicalizada por el anhelo estatal de control general de la producción y del mercado. En este contexto, sus objetivos específicos se relacionaron con "la solución para problemas sociales masivos, la reducción de la dependencia tecnológica y el desarrollo de la industria nacional" (Palmarola, 2002). Claramente, el contexto y los objetivos del Grupo estaban en sintonía con la teoría crítica de Ulm. En un contexto inestable, las condiciones establecidas por el programa político de Allende, permitieron definir en la práctica los horizontes profesionales del diseño en una sociedad no competitiva. Esto, dado que esta transferencia permitía sustituir los factores que según Abraham Moles, profesor de la escuela, habrían llevado a la Crisis del Funcionalismo:

La teoría económica de la sociedad acaudalada, sostiene que la maquinaria de la producción debe funcionar permanentemente. Es por este motivo que el consumidor debe ser estimulado para consumir a cualquier precio. El consumo y la producción están encadenadas en un sistema combinado que funciona a una velocidad cada vez mayor. El funcionalismo, necesariamente contradice la doctrina de la sociedad acaudalada, la cual es forzada a producir y a vender incesantemente. A fin de cuentas, el funcionalismo tiende a reducir el número de objetos y a alcanzar el calce óptimo entre productos y necesidades, mientras que la maquinaria productiva de la sociedad acaudalada fluye en la dirección opuesta. Esta crea un sistema de neokitsh a través de la acumulación de objetos en el medio ambiente humano. Y es en este punto donde la crisis del funcionalismo de hace evidente al estar dividido entre el neokitsh de supermercado y la consumación ascética de la función. (Moles, 1967, p.24)

El desprendimiento de la producción respecto del consumo -como consecuencia del control estatal sobre oferta y demanda- permitió sostener durante un período acotado, un enfoque funcionalista frente a los problemas 


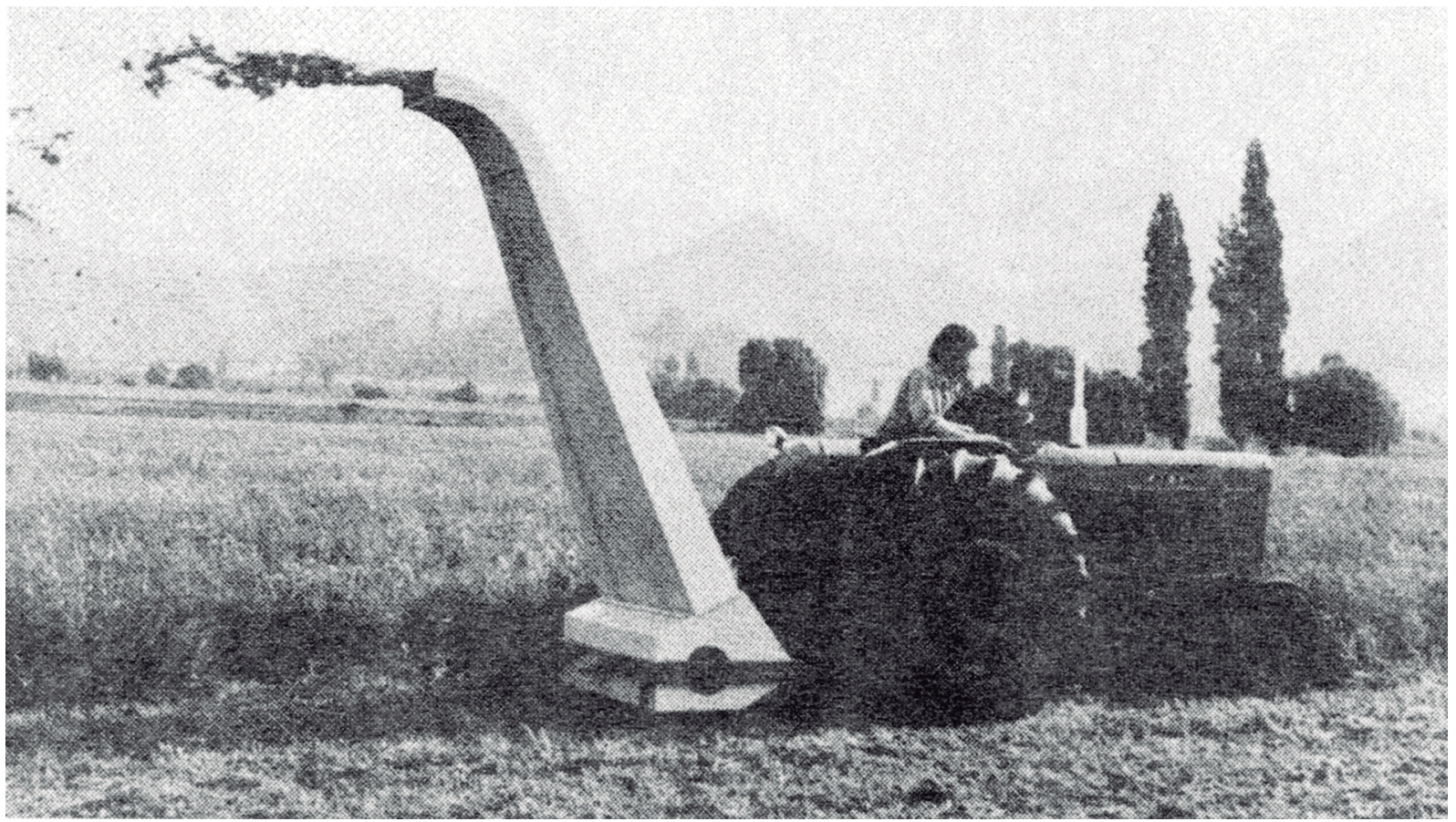

del diseño, en una práctica orientada socialmente, que buscó a la vez recuperar la estética como herramienta de liberación.

Esta experiencia fue finalmente interrumpida por el golpe de Estado de 1973, abortándose estos planes de desarrollo, y llevando a Bonsiepe a dejar el país poco después del 11 de septiembre. Sin embargo, el conjunto de proyectos desarrollados durante los 32 meses de trabajo del grupo, pueden ser considerados como vestigios de la implementación y transformación de este discurso crítico.

\section{El análisis de valor y la mejora del objeto técnico en una sociedad no competitiva}

Como resultados concretos de la transferencia del proceso metodológico de Ulm - a través del enfoque de Bonsiepe-, a las condiciones productivas posibilitadas por el gobierno de Allende, ${ }^{8}$ Ios proyectos desarrollados por INTEC se presentan como una serie de casos de estudio, en los cuales identificar las negociaciones políticas, metodológicas y estéticas del diseño en el contexto de una sociedad no competitiva. El campo de estas negociaciones fue la definición de metodologías propias al diseño industrial. Tal como afirmó Maldonado, la desvinculación del diseño y del marketing posibilitaron la revisión estructural y funcional de las propiedades técnicas del objeto, con el fin de "desestabilizar el carácter artificial y estable de los productos" (Maldonado, 1965).

Esta posibilidad -explorada en distintos proyectos- puede reconocerse con claridad en las distintas etapas del diseño de una segadora de follaje. Los criterios de diseño aplicados en cada una de estas etapas dan cuenta de la búsqueda de un nuevo rol para el diseñador en una sociedad no competitiva. Como muestra el registro de este proceso (Bonsiepe, 2010), la segadora fue desarrollada en dos intentos consecutivos, cada uno basado en una hipótesis de diseño distinta. En primer lugar, el grupo se centró en la "traducción" de una segadora de follaje extranjera a las capacidades de la plataforma
Figura 6. Segadora de follaje, INTEC, circa 1970. Fuente: Bonsiepe (2010)

8. Un registro de esta experiencia se presenta en dos iniciativas lideradas por Bonsiepe. La primera de ellas es la revista del INTEC, una publicación consistente en cuatro números desarrolladas durante la duración del Grupo de Diseño, y Design im Ubergang zum Sozialismus. Ein technish-politisher Erfahungs-berich aus dem Chile der Unidad Popular (1971-1973), una publicación de Bonsiepe de tres volúmenes, editada poco antes de su partida de su país. 
Figura 7. Plano segadora de follaje, mostrando el grosor del material, INTEC, circa 1970.

Fuente: Bonsiepe (2010).

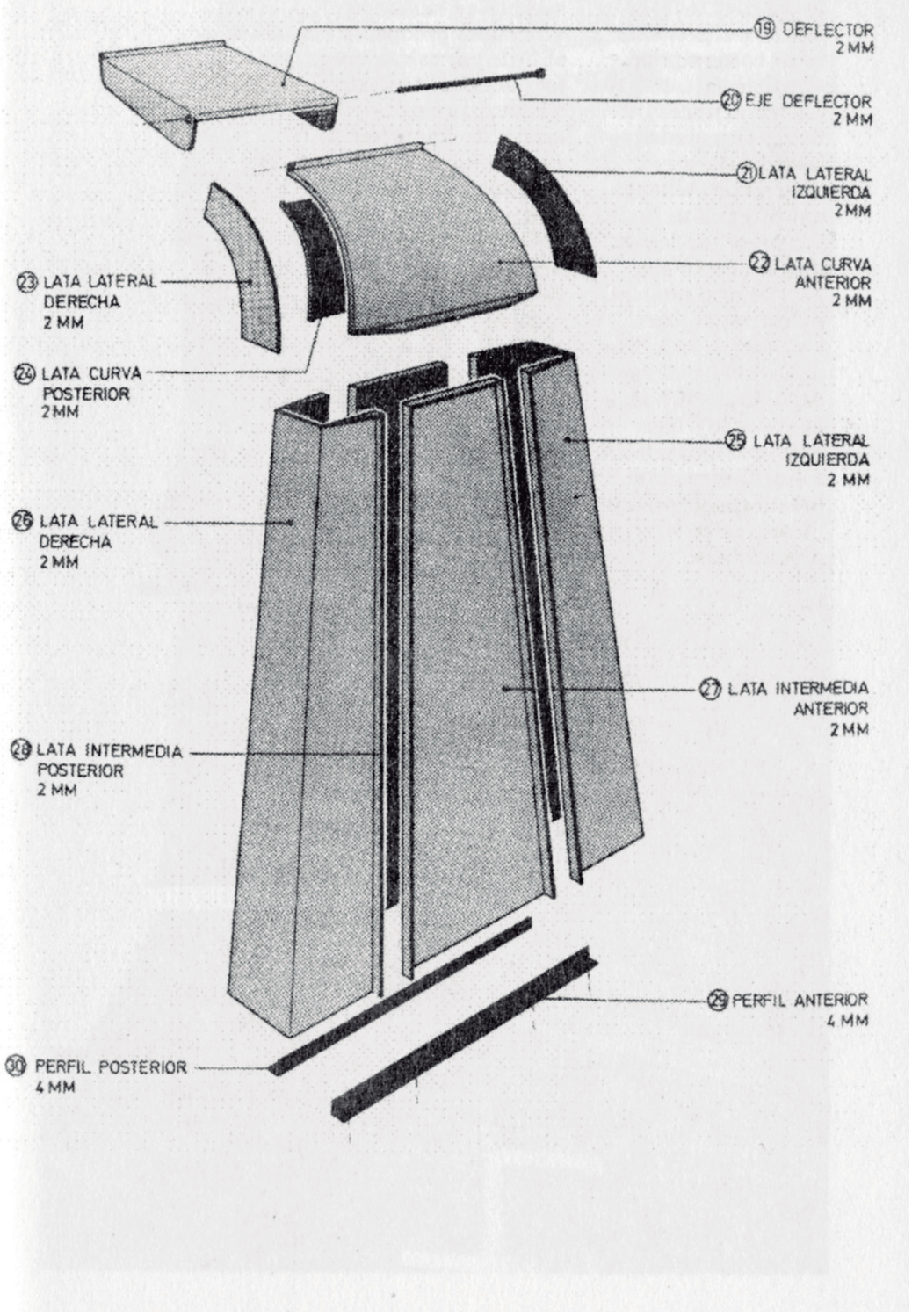

tecnológica instalada de Chile. Pero en función de este análisis se estimó que los modelos extranjeros, no eran lo suficientemente eficientes, tanto en términos de producción, como en términos de su desempeño.

Así, una segunda aproximación definió que aun cuando las relaciones geométricas del objeto original -entendidas como las características topológicas del objeto y, por lo tanto, independientes de sus características formales-, podían ser mantenidas, sus conceptos constructivos y formales debían ser revisados críticamente.

Esta aproximación hacia la geometría de la segadora tuvo como resultado la inclusión de un nuevo elemento en el diseño: un marco estabilizador que -actuando como contrapeso-, permitió la reducción del grosor del material, optimizando el peso de la segadora, su transportabilidad, costos 
de producción, demanda de material y consumo de combustible, además de reducir el proceso productivo y la cantidad de piezas.

Este proceso que "redujo la complejidad de la máquina en un $40 \%$ " (Bonsiepe, 2010, p.8), puede ser visto como un ejemplo práctico de la aplicación del análisis de valor, método discutido por Bonsiepe en Arabesques of Rationality, dentro de su análisis de los métodos científicos adecuados por la práctica del diseño:

El análisis de valor es una técnica industrial moderna que analiza críticamente la proporción entre utilidad y costo, propia de los elementos que conforman los productos. Esta técnica comparte su aproximación con el tipo de procedimientos que los diseñadores aplican o deberían aplicar. Está dirigido hacia la función, en vez de hacia el elemento. Su preocupación principal es no es el objeto - las formas estables- sino la función. En comparación con el procedimiento estándar de reducción de costos (el reemplazo de un componente por otro más barato, sin alterar el producto), el análisis de valor se diferencia en que cuestiona la estructura misma del producto, es decir, no considera la estructura del producto como precedente inalterable. Cada componente es evaluado en términos de lo que hace (su función), y en términos de cuanto cuesta esta función. Esto implica observar el producto como un conjunto de "generadores de utilidad": cada "generador de utilidad" cuesta dinero; su costo puede compararse contra su desempeño, y lo que se intenta hacer es maximizar la utilidad, al mismo tiempo que se reduce en forma relativa el costo, o en términos generales, esforzarse por la optimización del valor. El valor, es determinado por la proporción entre costo y utilidad (o entre input y output). (Bonsiepe, 1967, p.19)

La forma en la cual el análisis de valor es aplicado en el proceso de diseño de la segadora permite identificar el tipo de conocimiento que debe ser considerado por el diseñador profesional en una sociedad no competitiva. Además, permite evaluar la pertinencia de la aplicación de este método en el contexto de este tipo de sociedad.

En este sentido, esta acción permite revisar el cuestionamiento de la relación entre funcionalismo y consumo, dado que este modo de optimización estructural no sería posible en una sociedad competitiva: dado el incremento de su valor de uso y la reducción de su costo de producción, su valor de cambio sería puesto en riesgo, dado que su competitividad sería apoyada por procesos productivos establecidos, ciclos de obsolescencia y criterios comerciales.

\section{Styling, anti-styling y diseño de productos}

Dentro de este análisis de los diferentes objetos diseñados por INTEC, un proyecto presenta puntos críticos para la interpretación de las negociaciones del diseño en una sociedad no competitiva, dado que su valor de uso no está directamente ligado a necesidades básicas, ni a la optimización de su producción, sino al consumo. En este sentido, la preponderancia del factor estético en el diseño del tocadiscos portátil desarrollado por el Grupo, lo hace merecedor de un análisis más detenido.

En primer lugar, el registro de su proceso de diseño, pero más aun, su lugar en el programa del INTEC, es contradictorio. Por un lado, el registro de un 


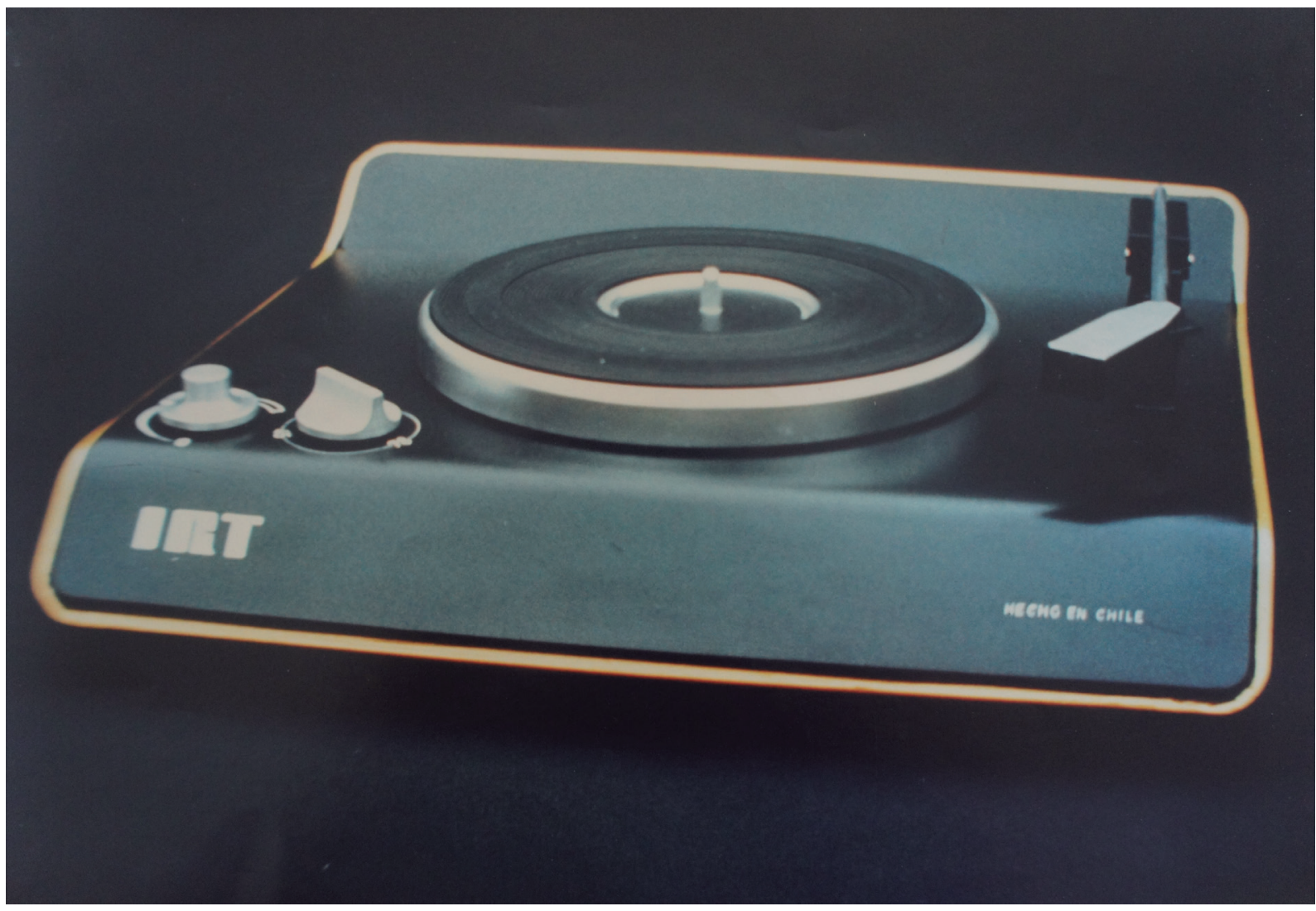

Figura 8. Prototipo de un tocadiscos portátil, INTEC, circa 1971.

Fuente: Archivo de Rodrigo Walker.

9. La cita del pasaje corresponde a una imagen, escrita, presumiblemente, por el editor del libro. comentario de Bonsiepe afirma que era "injustificable trabajar en un producto así de lujoso, en tiempos de escasez de alimento y en contraste con el programa sociopolítico de la Unidad Popular" (Bonsiepe, 2010, p.20). 9 Por otro, una estrategia de persuasión de parte del Grupo hacia el gobierno insinuaba que "ellos estratégicamente argumentaron que el tocadiscos portátil estaba pensado para absorber el capital de la burguesía para proyectos más importantes y así evitar la inflación" (Bonsiepe, 2010, p.20).

Lo cuestionable de este argumento (los recursos humanos del INTEC vs. los beneficios del negocio pueden no ser lo suficientemente amplios para evitar la inflación), se opone al estudio de Medina quien, al abordar el alcance de la revolución en Chile afirma que:

La revolución chilena tomo lugar en muchos frentes [...], en los laboratorios, tableros de dibujo y líneas de producción. Esto transformó la manera en la que la investigación científica era realizada, así como también, el alcance de proyectos públicos de ingeniería y economía. Por ejemplo, este tocadiscos fue construido por el primer grupo de diseño financiado desde el Estado, formado durante el gobierno de Allende. La idea era tomar un bien que anteriormente solo había sido accesible para las élites adineradas y hacerlo accesible a las masas. (Miller Medina, 2009)

Como estas referencias postulan, es imposible posicionar al tocadiscos portátil simultáneamente como un producto para obtener ganancias desde las 


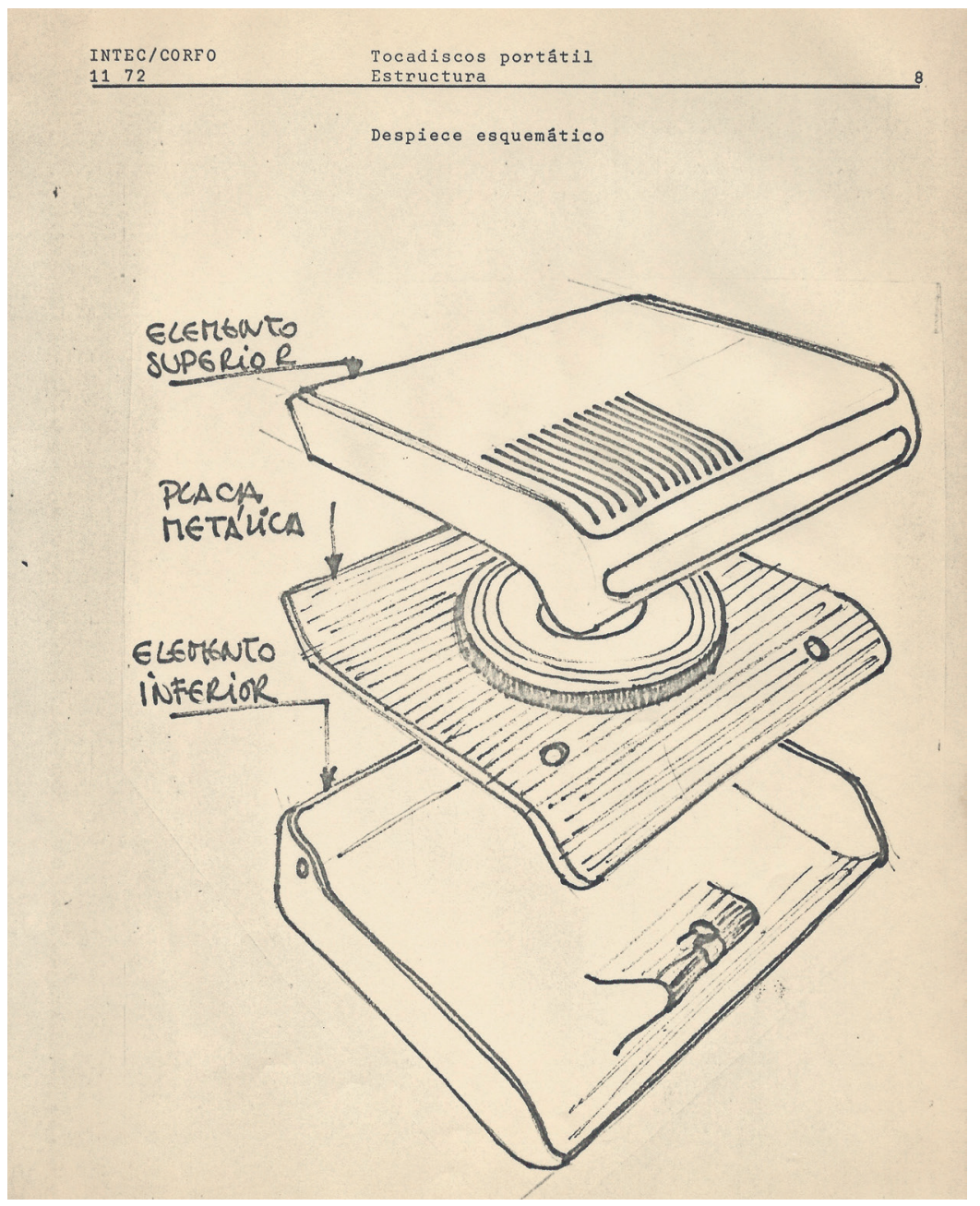

Figura 9. Despiece esquemático, INTEC, circa 1971. Fuente: Archivo de Rodrigo Walker.

clases acomodadas y como un agente de democratización del diseño. En ese sentido, queda claro que su interpretación como objeto cultural no está de acuerdo con los factores culturales de su diseño.

Lo que sí puede afirmarse a partir de la información disponible, es que este prototipo es el resultado de la integración de factores estéticos y productivos. Los aspectos productivos considerados en su proceso de diseño son posibles de reconocer en el tratamiento simétrico que se le dio a los componentes del gabinete del tocadiscos, decisión que puede ser inferida como resultado de la aplicación de análisis de valor. Los factores estéticos pueden ser vistos en el diseño como el estilo que rompe esa simetría.

El tocadiscos portátil habita en una zona intermedia entre estos dos polos que refiere Bonsiepe:

El estilista que hermosea la superficie para excitar el apetito de prestigio por parte del consumidor, es rechazado con desesperación como un "cosmetólogo de productos". El diseñador, se supone, está interesado en temas más serios: su interés está en el concepto del producto, en el mejoramiento de sus características de uso, en la facilidad de su ensamblaje, en la reducción 
de su costo de producción, en resumen, está interesado en satisfacer necesidades reales. Él produce un diseño apropiado, y no un diseño "de prestigio", como el estilista [...] Ahora, sería un serio error el pensar que descartar del diseño la filosofía del styling abriría mágicamente el camino para alcanzar la calidad formal y funcional en el diseño [...] Las dudas acerca del procedimiento y la filosofía de diseño del estilista, están totalmente confirmadas pero, por otro lado, una estrategia que prácticamente elimine la responsabilidad sobre la forma de un producto, 0 que la reduzca a la mera coordinación de factores de diseño, tampoco puede contemplarse. Mientras que la imaginación estética del estilista tiende a la hipertrofia, el antiestilismo tiende a la atrofia. (Bonsiepe, 1967, pp.11-12)

\section{Conclusiones}

Alexander plantea que un problema de diseño debe enfrentarse como uno compuesto por dos elementos, una forma y un contexto que le pertenece. El contexto -en gran parte identificable con la suma de sus requerimientos y limitantes-, recibe su complemento racional a través de una forma adecuada a él, mientras que la forma encarna la suma de las características que satisfacen al contexto. Una forma es apropiada a un contexto si esta es conducente a la fluida coexistencia de ambos. Dado que cada vez es más difícil - si no imposible-describir una forma que se adecúa a su contexto (por ejemplo, una silla cómoda), es preferible enumerar los posibles tipos de descalce entre forma y contexto. De esta forma, el proceso de diseño puede ser interpretado como una estrategia diseñada para neutralizar, eliminar y erradicar los factores que causan estos descalces no deseados entre forma y contexto. De hecho, solo contamos con un problema de diseño cuando un descalce - un estado de deficiencia - es reconocido.(Bonsiepe, 1967, p.17)

Transfiriendo estos comentarios de Bonsiepe acerca de las ideas de Alexander, desde el proceso de diseño hacia la evaluación de la experiencia del Grupo de Diseño del INTEC, es posible argumentar que en la investigación a desarrollar, serán este tipo de calces y descalces observables en los proyectos llevados a cabo por el Grupo, los que darán cuenta de la forma en la que la teoría crítica de $\mathrm{HfG}$-Ulm pudo realizarse desde su implementación temporal en una sociedad no competitiva.

Esta transferencia productiva implicó reconocer el grado de desprendimiento posible entre producción y consumo, en función de su contexto de aplicación. Preliminarmente, podemos concluir que mientras por un lado se logró ejercitar un enfoque racionalista hacia el diseño de productos - a través de la mejora fundamental de los objetos técnicos, por medio del método de análisis de valor-, por otro lado el peso específico de las variables estéticas y su rol semántico en relación a su consumo y su función, es ambiguo, aun cuando intenten mantenerse en un "desapego inmaculado y apolítico respecto de lo social” (Bonsiepe, 1968, p.16). 


\section{Bibliografía}

Bonsiepe, G. (1967). Arabesques of Rationality. Notes on the Methodology of Design. Journal OfThe Ulm School of Design, (19-20), 9-23.

Bonsiepe, G. (1968). A Method for Quantifying Order in Typographical Design. Journal of the UIm School of Design, (21), 24-31.

Bonsiepe, G. (1968). Commentary on the Situation of the HfC. Journal of the UIm School of Design, (21), 5-14.

Bonsiepe, G. (1968). Communication and Power. Journal of the Ulm School of Design, (21), 16-20.

Bonsiepe, G. (1968). End of the Journal Ulm. Journal of the Ulm School of Design, (21), 4.

Bonsiepe, G. (1968). Semantic Analysis. Journal of the UIm School of Design, (21), 33-37.

Bonsiepe, G. (2006). El discurso de Ulm. En D. Rinker, M. Quijano \& B. Reinhardt, Modelos de Ulm - modelos post-UIm | hochschule für gestaltung UIm 1953-1968 (pp. 15-17). Stuttgart: Hfc-Archiv/Ulmer Museum.

Bonsiepe, G. (2010). Design im Übergang zum Sozialismus, 1974 (Excerpt) / Design in Transition to Socialism. A Techno-Political Field Report from Unidad Popular's Chile (1971-1973). En Civic City Cahier 2: Design and Democracy (pp. 5-27). London: Bedford Press.

Corfo | Corporación de Fomento de la Producción. (2010). Historia de CORFO - Sueños de futuro IV. Recuperado de https://youtu.be/ANRowVcsVq8 (12 de Mayo de 2011). Fernández, S. (2006). La influencia de la Hfc Ulm, en la enseñanza de diseño en América Latina. En D. Rinker, M. Quijano \& B. Reinhardt, Modelos de Ulm - modelos post-UIm | hochschule für gestaltung UIm 1953-1968 (pp. 18-21). Stuttgart:HfG-Archiv/Ulmer Museum.

Frampton, K. (1974). Apropos Ulm. Oppositions, (3), 17-36. Kepes, G. (1965). Education of vision. New York: G. Braziller. Lindinger, H. (1991). Ulm Design. The Morality of Objects.

Cambridge, Mass.: MIT Press.

Maldonado, T. (1965). Design Education. En G. Kepes, The Education of Vision (pp. 122-135). New York: G. Braziller. Miller Medina, J. (2005). The State Machine: Politics, ideology and Computation in Chile, 1964-1973 (Ph.D.). History and Social Study of Science and Technology, MIT.

Miller Medina, J. E. (2009). Cybernetics and Revolution. MediaBerkman, Berkman Center for Internet of Society. Recuperado de http://blogs.harvard.edu/ mediaberkman/2009/03/04/cybernetics-andrevolution-eden-medina/ (12 de Mayo de 2011).

Moles, A. (1967). Functionalism in Crisis. Journal of The UIm School of Design, (19-20), 24-25.

Ortega, L., Norambuena, C., Pinto, J., \& Bravo, G. (1989). CORFO, 50 años de realizaciones. Santiago de Chile: Departamento de Historia USACH.
Palmarola, H. (2002). Diseño Industrial Estatal en Chile 1968-1973. Conferencia presentada el 12 de Noviembre al ciclo Testimonios de la Modernidad. Facultad de Arquitectura, Diseño y Estudios Urbanos, Pontificia Universidad Católica de Chile, Santiago de Chile. Recuperado de http://www.guibonsiepe.com/pdffiles/ timeline_design_chile.pdf (12 de Mayo de 2011).

Resolution of the Hochschule für Gestaltung. (1968). Journal OfThe Ulm School Of Design, (21), 15.

Schnaid, C. (1967). Architecture and Political Commitment. Journal OfThe UIm School Of Design, (19-20).

Spitz, R. (2002). hfg ulm. The View Behind the Foreground. The Political History of the UIm School of Design, 19531968. Stuttgart: Edition Axel Menges. 